\title{
Demodicose suína no norte do estado do Rio de Janeiro: relato de caso
}

\author{
[Demodicosis in northern Rio de Janeiro State: case report] \\ R.L. Silveira ${ }^{1}$, R.B. Ribeiro ${ }^{2}$, A.C.M. Cruz ${ }^{3}$, L.G. Almeida ${ }^{4}$, E.C.Q. Carvalho ${ }^{4}$ \\ ${ }^{1}$ Aluno de pós-graduação - Universidade Estadual do Norte Fluminense Darcy Ribeiro (UENF) \\ Campos dos Goytacazes, RJ - Docente - Universidade Federal Fluminense (UFF) - Niterói, RJ \\ ${ }^{2}$ Aluna de pós-graduação - UENF - Campos dos Goytacazes, RJ \\ ${ }^{3}$ Universidade Federal Fluminense (UFF) - Niterói, RJ \\ ${ }^{4}$ CCTA - UENF - Campos dos Goytacazes, RJ
}

\begin{abstract}
RESUMO
Um leitão oriundo de um criatório do norte do estado do Rio de Janeiro, mantido no biotério do Hospital Veterinário da Universidade Estadual do Norte Fluminense Darcy Ribeiro e utilizado como caso piloto de projeto de pesquisa para investigações sobre o acesso videolaparoscópico retal (NOTES - Natural Orifice Translumenal Endoscopic Surgery), foi eutanasiado e necropsiado. Amostras do ponto de acesso do tubo de inserção na região mucocutânea anal foram colhidas, fixadas em formalina neutra tamponada a $10 \%$, processadas por inclusão em parafina e coradas pela hematoxilina e eosina para histologia. Observou-se o comprometimento de folículos que, dilatados, exibiam formações que lembravam Demodex spp. cortados em vários sentidos e, por se tratar de ácaros espécie-específicos, concluiu-se como D. phylloides. Na citopatologia, o ácaro foi recuperado e identificado a fresco, mostrando-se alongado, com quatro pares de patas ventrais e no terço anterior do corpo. Este se refere ao primeiro registro de demodicose suína no estado do Rio de Janeiro.
\end{abstract}

Palavras-chave: suíno, demodicose, Demodex phylloides

\begin{abstract}
A piglet derived from a farm in northern Rio de Janeiro State, maintained at the Animal Veterinary Hospital of Universidade Estadual do Norte Fluminense Darcy Ribeiro and used as a pilot case for a research project on access to research video-laparoscopic rectal (NOTES - Natural Orifice Endoscopic Surgery Translumenal) was euthanized and necropsied. Samples from the access point of the insertion tube in mucocutaneous anal region were collected, fixed in buffered formalin 10\%, processed by embedding in paraffin and stained with hematoxylin and eosin for histopathology. The impairment of follicles that exhibited dilated formations resembling Demodex spp. cut in various directions and due to the mite species-specific was observed, and was concluded as $\mathrm{D}$. phylloides. The mite was recovered and freshly identified by cytopathology. It was elongated, with four pairs of legs, at ventral and anterior third of the body. This refers to the first report of swine demodicosis in Rio de Janeiro State.
\end{abstract}

Keywords: swine, demodicosis, Demodex phylloides

\section{INTRODUÇÃO}

Os ácaros Demodex spp. infestam os folículos pilosos e as glândulas sebáceas de todas as espécies de animais domésticos, sendo espécieespecíficos (Smith, 2006; Radostits et al., 2002). Em circunstâncias normais, causam danos mínimos e, em alguns casos, coabitam como comensais (Wall, 2007). Ao contrário da sarna sarcóptica, que afeta a eficiência produtiva (Arends et al., 1990; Vercruysse e Smets, 2000) e implica importantes perdas econômicas (Galuppi et al., 2007), além de apresentar alta prevalência em vários países (Cargill e Jackowiak, 2003) e ser o mais comum

Recebido em 1 de novembro de 2011

Aceito em 10 de janeiro de 2012

E-mail: renatosilveira@vm.uff.br 
ectoparasita dos suínos (Baker, 2005), a sarna demodécica é relativamente rara (Doster, 1995) e causada pelo Demodex phylloides. O ácaro é alongado $(0,23-0,26 \mathrm{~mm})$ e possui quatro pares de patas curtas. Vive permanentemente como parasita dos suínos, onde faz todo o seu ciclo, o qual, admite-se, completa-se em 20-35 dias. O parasita adulto pode viver de um a dois meses no hospedeiro. Fora dele, não mais que dois dias (Sobestiansky et al., 1999).

Segundo McGavin e Zachary (2009), o agente produz pápulas cobertas de escamas, que progridem para nódulos preenchidos com detritos queratináceos e ácaros, localizando-se preferencialmente na pele da região ventral, pálpebras e nariz.

Os dados sobre a distribuição e a incidência da enfermidade são raros na literatura científica (Santarém et al., 2005), apesar de ter sido diagnosticada em vários países, como: Austrália, Estados Unidos, Quênia, Nova Zelândia, vários países europeus e ilhas do Pacífico (Cargil e Davies, 2006). Muitas vezes, a doença cursa sem sinais clínicos, fazendo com que a maior parte dos registros de sua ocorrência fique restrita aos achados de inspeção nos frigoríficos (Sobestiansky e Barcellos, 2007).

A transmissão do ácaro dá-se, provavelmente, pelo contato direto dos animais, embora seja difícil a reprodução artificial da parasitose (Nutting, 1976), que é, frequentemente, subclínica (Nutting et al., 1975).

O diagnóstico é baseado na sintomatologia clínica e confirmado pela identificação do parasita ao exame microscópico do material colhido das lesões. Adicionalmente, pode ser feita biópsia das áreas lesadas, a qual revelará folículos pilosos distendidos, em diversos graus, contendo os ácaros demodécicos. Muitas vezes se identificam grandes cistos foliculares com mínima resposta inflamatória. Alternativamente, variados graus de foliculite, perifoliculite, furunculose e formação de granulomas, do tipo corpo estranho, podem ser identificados (Sobestiansky e Barcellos, 2007). Segundo Cargil e Davies (2006), a confirmação do diagnóstico é feita pela demonstração do
Demodex phylloides recuperado das lesões. Nos casos severos, o animal deve ser descartado.

O objetivo deste trabalho foi registrar, mediante achados histopatológicos e acaroscópicos, a ocorrência de demodicose suína no norte do estado do Rio de Janeiro.

\section{CASUÍSTICA}

Um leitão oriundo de um criatório do norte do estado do Rio de Janeiro, mantido no biotério do Hospital Veterinário da Universidade Estadual do Norte Fluminense Darcy Ribeiro, foi utilizado em caso piloto de projeto de pesquisa para acesso vídeolaparoscópico retal (NOTES - Natural Orifice Translumenal Endoscopic Surgery). Amostras do ponto de acesso do tubo de inserção na região mucocutânea anal foram colhidas e fixadas em formalina neutra tamponada a $10 \%$. Em seguida, foram processadas por inclusão em parafina e coradas pela hematoxilina e eosina para avaliação histopatológica. Impressionou nas amostras da região o comprometimento de folículos que, dilatados, exibiam formações que lembravam Demodex phylloides, cortados em vários sentidos associados a escassos mononucleares e neutrófilos (Fig. 1). Na citopatologia, o ácaro foi recuperado $\mathrm{e}$ identificado a fresco, mostrando-se alongado e, conforme Jing et al. (2005), com quatro pares de patas ventrais (podosoma) no terço anterior do corpo (Fig. 2).

\section{DISCUSSÃO}

Os ácaros vivem nos folículos pilosos e produzem de leve a moderada dermatite sobre o focinho, na face medial das coxas e ao longo dos flancos, eventualmente podendo acometer todo o corpo, levando à condenação da carcaça no matadouro (Doster, 1995). Na maioria das vezes, apresentam-se sem sinais evidentes (Santarém $e t$ al., 2005). No presente estudo, o ácaro foi demonstrado na região mucocutânea anal e, também, sem sinais clínicos. Em relato de caso em bovino, subclínico, Lemos et al. (2005) observaram lesões perivulvares representadas por pequenas nodulações com espessamento cutâneo, cujas secções microscópicas evidenciaram ácaros isolados ou em grupos no interior de folículos pilosos. 


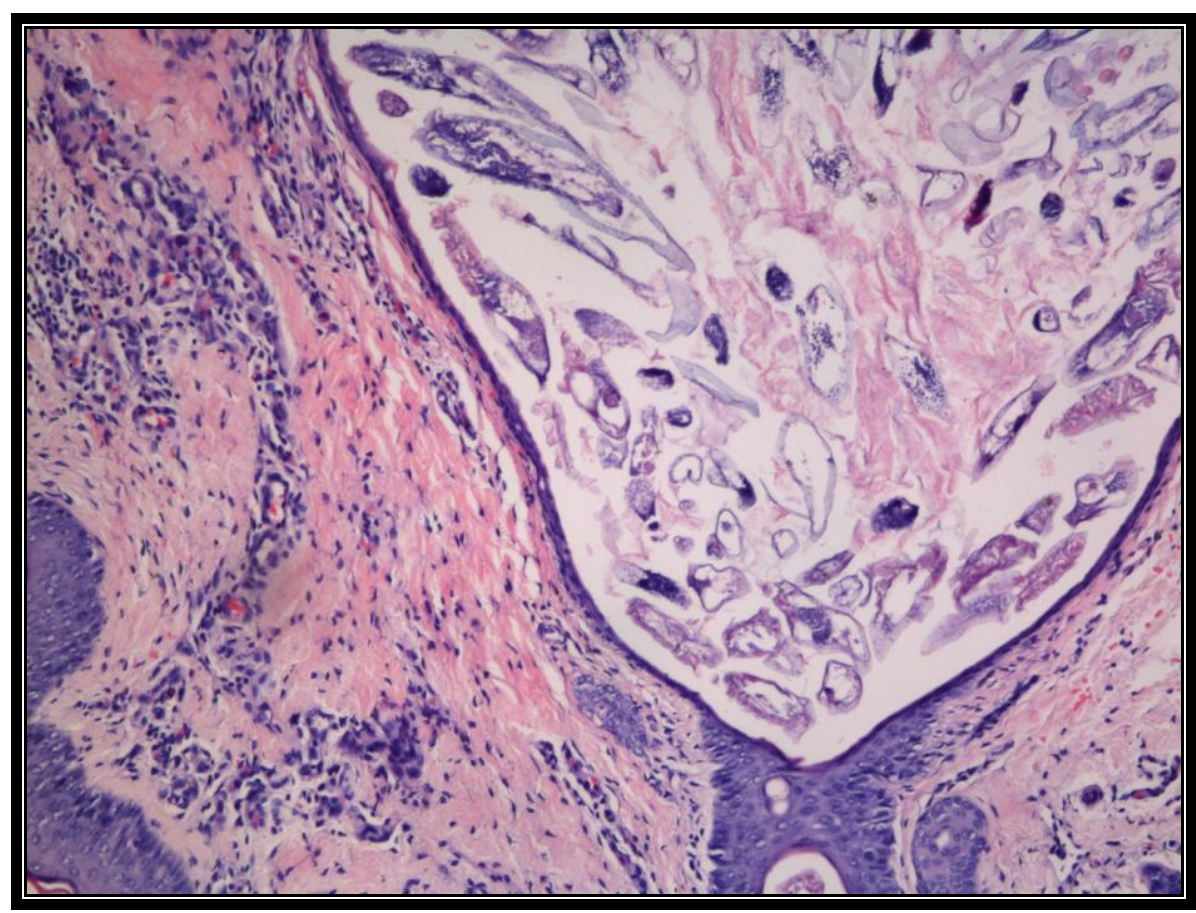

Figura 1. Pele de suíno. Demodicose. Folículo piloso dilatado e com formações que lembram os Demodex phylloides, associados a mononucleares e neutrófilos. HE. Objetiva $20 \mathrm{X}$.

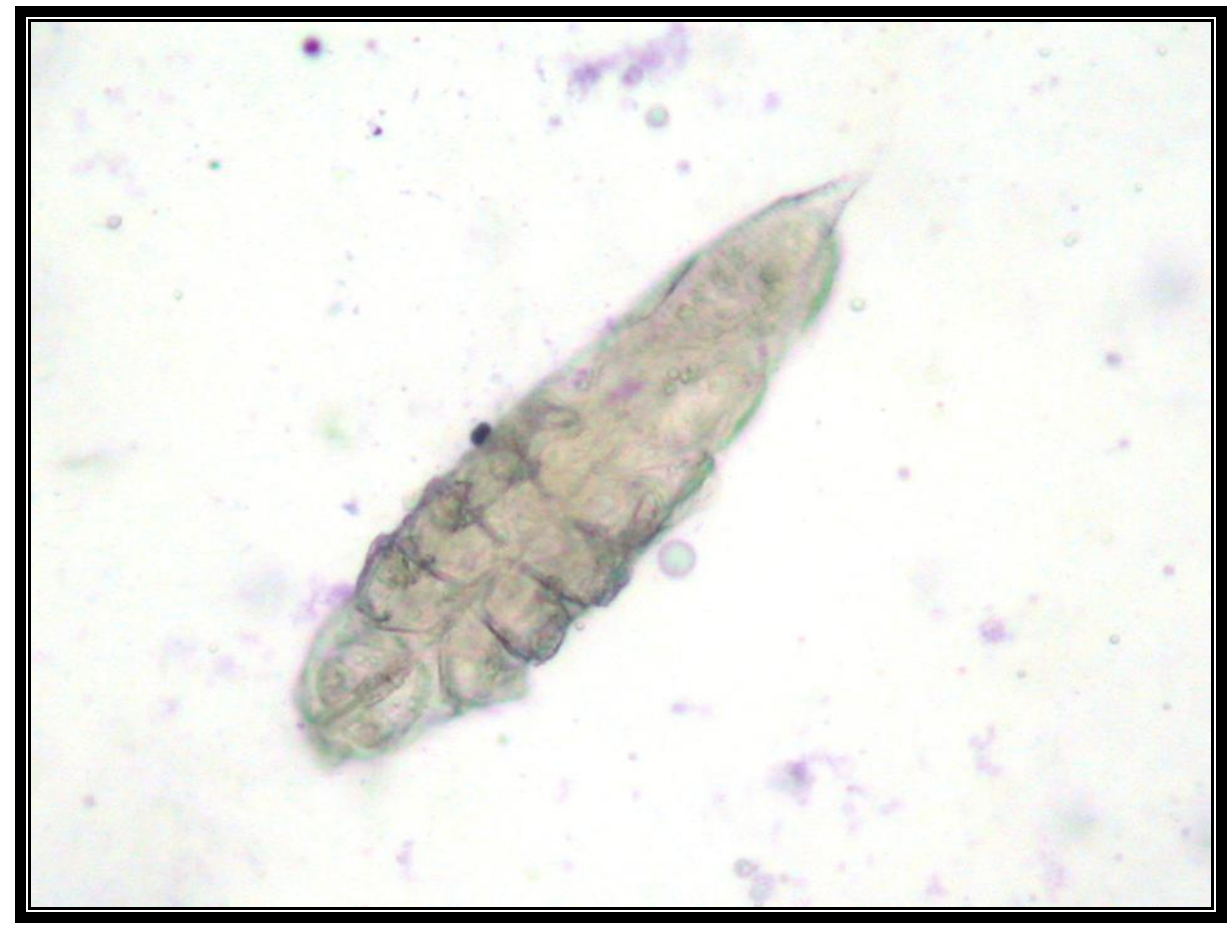

Figura 2. Preparação a fresco de um espécime colhido de caso de foliculite cutânea correspondendo ao Demodex phylloides. Objetiva $20 \mathrm{X}$. 
Foram observadas, como descrito também por Santarém et al. (2005), em exame histopatológico, numerosas formas intrafoliculares do ácaro. Neste caso, sem ruptura folicular, a resposta inflamatória foi mínima, não granulomatosa e caracterizada por raros neutrófilos. Mendes et al. (2007) observaram hiperplasia dos folículos pilosos, que continham no seu interior material necrótico, fragmentos de parasitas, grumos de bactérias na forma de cocos e células inflamatórias mononucleares, bem como intensa fibrose perifolicular, provavelmente decorrente de uma complicação pela ruptura folicular. Em bovinos, Lemos et al. (2005) observaram hiperqueratose e foliculite, representada por infiltrado misto rico em neutrófilos.

Ainda se observa a condição epidemiológica desta parasitose quanto ao seu caráter emergente ou negligenciado.

\section{REFERÊNCIAS}

ARENDS, J.J.; STANISLAW, C.M.; GERDON, D. Effects of sarcoptic mange on lactating swine and growing pigs. J. Anim. Sci., v.68, p.14951499. 1990.

BAKER, R.B. Skin and other miscellaneous diseases. In: Proceedings of the North Carolina Health Hogs Seminar. North Carolina. 2005. http://www.ncsu.edu/project/swine_extension/he althyhogs/book2005/baker/baker_skin.pdf. Acessado em 5 Outubro, 2010.

CARGIL, C.; DAVIES, P.R. External parasites. In: (STRAW, B.E; ZIMMERMAN, J.J.; D'ALLAIRE; TAYLOR, D.J.) Diseases of swine. 9.ed. Ames. Blackwell Publishing, 2006. Cap. 53, p.875-889.

CARGIL, C.; JACKOWIAK, J. Mange monitoring makes economic sense. Pig Progress. v.19, p.27-28, 2003.

DOSTER, A.R. Skin diseases of swine. Swine Health Prod., v.3, p.256-261. 1995.

GALUPPI, R.; AVENOSO, A.M.; LEOTTI, G. et al. Diagnosis of Sarcoptic Mange in slaughtered swine. Vet. Res. Communications, v.31, Suppl. 1, p.233-236. 2007.
JING, X.; SHULING, G.; YING, L. Environmental scanning electron microscopy observation of the ultrastructure of Demodex. Microsc. Res. Technique,v.68, p.284-289, 2005.

LEMOS, L.S.; SANTOS, A.S.O.; SALES, L.G. et al. Demodicose bovina: relato de caso. Arq. Bras. Med. Vet. Zootec., v.57, p.732-735, 2005.

McGAVIN, M.D.; ZACHARY, J.F. Bases da Patologia e Veterinária. 4.Ed. Rio de Janeiro: Elsevier, 2009. 1476p,

MENDES, M.C.; DEL FAVA, C.; SPÓSITO FILHA, E. et al. Ocorrência de demodicose em suínos da periferia da cidade de São Paulo. Arq. Inst. Biol., v.69, p.143, 2007.

NUTTING, W.B. Hair follicle mites (Demodex spp.) in New Zealand. New Zealand J. Zool., v.2, p.219-222, 1975.

NUTTING, W.B. Hair follicle mites (Demodex spp.) of medical and veterinary concern. Cornell Vet., v.66, p.214-231, 1976.

RADOSTITS, O.M.; GAY, C.C.; BLOOD, D.C.; HINCHCLIFF, K.W. Clínica Veterinária: Um Tratado de Doenças dos Bovinos, Ovinos, Suínos, Caprinos e Equinos. 9.Ed. Rio de Janeiro. Guanabara koogan. 1737p. 2002.

SANTARÉM, V.A.; FARIAS, M.R.; TOSTES, R.A. Demodectic mange in fattening pigs in São Paulo, Brazil. Vet. Parasitol., v.131, p.169-171, 2005.

SMITH, B.P. Medicina Interna de Grandes Animais. 3.Ed. Barueri: Manole, 2006. 1728p.

SOBESTIANSKY, J.; BARCELLOS, D.E.S.N. Doenças dos suínos. Goiânia: Cânone Editorial, 2007. 768p,

SOBESTIANSKY, J.; BARCELLOS, D.E.S.N.; MORES, N. et al. Clínica e Patologia suína. Goiânia: Gráfica, 1999. Art 3. 464p,

VERCRUYSSE, J.; SMETS, K. The diagnosis of swine mange: a European perspective. In: INTERNATIONAL PIG VETERINARY SOCIETY (IPVS) Congress, 16., 2000, Melbourne. Proceedings... Melbourne: Editora, 2000. p.266.

WALL, R. Ectoparasites: Future challenges in a changing world. Vet. Parasitol., v.148 p.62-74. 2007. 